\title{
$\beta$-Carotene nanodispersions: preparation, characterization and stability evaluation
}

\begin{abstract}
The aim of the present study was to investigate the preparation of b-carotene nanodispersions as potential active ingredients for food formulations. Nanodispersions containing 6 -carotene were obtained by a process based on an emulsificationï evaporation technique. The preparation method consisted of emulsifying an organic solution of b-carotene in an aqueous solution containing emulsifier using two different homogenizers (a conventional homogenizer and a microfluidizer), followed by direct solvent evaporation under reduced pressure. The influence of different homogenizing conditions (pressure and cycle) and two organic/aqueous phase ratios on particle size parameters and content of $\tilde{6}$-carotene was investigated. In addition, the stability of b-carotene nanodispersions was carried out at a storage temperature of $4{ }^{\circ} \mathrm{C}$. The particle size distribution of $\mathrm{b}$-carotene in nanodispersions was demonstrated with a laser diffraction particle size analyzer and the retention of $b$ carotene in the prepared nanodispersions was studied by high-pressure liquid chromatography. In general, homogenization pressure and cycle had significant $(\mathrm{P}<0.05)$ effects on various particle size parameters. A volume-weighted mean diameter (D4,3) of $\mathrm{b}$ carotene nanoparticles, ranging from 60 to $140 \mathrm{~nm}$, was observed in this study.
\end{abstract}

Keyword: Emulsification-evaporation; b-carotene; Nanodispersion; High-pressure homogenization; Particle size analysis 